Rockefeller University

Report No. DOE/ER/40033-20

(RU 81/A-19)

$E-396$

\title{
UNIVERSALITY OF CHARGED MULTIPLICITY DISTRIBUTIONS
}

K. Goulianos

The Rockefeller University, New York, N.Y. 10021

Presented at

The 2nd Topical Conference on Forward Collider Physics

Madison, Wisconsin

December 1981 


\title{
UNIVERSALITY OF CHARGED MULTIPLICITY DISTRIBUTIONS
}

\author{
K. Goulianos*
}

The Rockefeller University, New York, N.Y. 10021

\begin{abstract}
The charged multiplicity distributions of the diffractive and non-diffractive components of hadronic interactions, as well as those of hadronic states produced in other reactions, are described well by a universal Gaussian function that depends only on the available mass for pionization, has a maximum at $n_{0} \cong 2 M^{\frac{1}{2}}$, where $M$ is the available mass in $\mathrm{GeV}$, and a peak to width ratio $\mathrm{n}_{\mathrm{o}} / \mathrm{D} \cong 2$.
\end{abstract}

\section{INTRODUCTION}

This report consists of two parts. In the first part, we present the results of a measurement of charged multiplicity distributions of high mass diffractive $\pi^{ \pm}, \mathrm{K}^{ \pm}$and $\mathrm{p}^{ \pm}$states produced in 100 and 200 $\mathrm{GeV} / \mathrm{c}$ hadron-proton collisions, $\mathrm{h}+\mathrm{p} \rightarrow \mathrm{X}+\mathrm{p}$. We find that these distributions are described well by a Gaussian function that depends only on the mass available for pion production, $M \cong M_{x}-M_{h}$, peaks at $\mathrm{n} \cong 2 \mathrm{M}^{\frac{1}{2}}$, where $\mathrm{M}$ is in $\mathrm{GeV}$, and has a peak to width ratio $n_{0} / D^{0} \cong 2$, where $D=\left(n^{2}-n_{0}{ }^{2}\right)^{\frac{1}{2}}$. The independence of the charged multiplicities from the quantum numbers of the diffractive state led us to examine whether the multiplicities of hadronic matter in general also follow the same distribution function. In the second part of this report, we show that this is Indeed the case.

\footnotetext{
* The work presented in this report was done by the following Rockefeller University groups:

(a) PART I : R.L. Cool, K. Goulianos, S.L. Segler ${ }^{+}$, H. Sticker and S.N. White

(b) PART II : K. Goulianos, H. Sticker and S.N. White

+ Present address: Fermi National Accelerator Laboratory Batavia, Illinois 60510
} 
PART I

CHARGED MULTIPLICITIES
OF HIGH MASS DIFFRACTIVE $\pi^{ \pm}, \mathrm{k}^{ \pm}$and $\mathrm{p}^{ \pm}$STATES

The charged multiplicities of the diffractive states $\mathrm{X}$ produced in the reaction

$$
h+p \rightarrow x+p\left(h=\stackrel{ \pm}{\pi^{ \pm}}, k^{ \pm}, p^{ \pm}\right)
$$

were measured recently in experiment E-396 at Fermilab. Reaction (1) was studied at incident beam momenta of 100 and $200 \mathrm{GeV} / \mathrm{c}$ in the kinematic range $0.025<|t|<0.095(\mathrm{GeV} / \mathrm{c})^{2}$ and $1-\mathrm{x} \cong\left(\mathrm{M}_{\mathrm{x}}{ }^{2}-\mathrm{M}_{\mathrm{h}}{ }^{2}\right) / \mathrm{s}<0.1$, where $x$ is the Feynman scaling variable, $x=p_{11} / p_{11}$, max $\cdot$ Results from this experiment on elastic scattering and on the $M{ }^{2}$ dependence and factorization properties of diffraction dissociation have already been published $[1,2]$. The results on charged multiplicity distributions reported here are new.

The experiment was performed in the M6W beam line of the Meson Laboratory. A plan view of the apparatus is shown in Figure 1 .

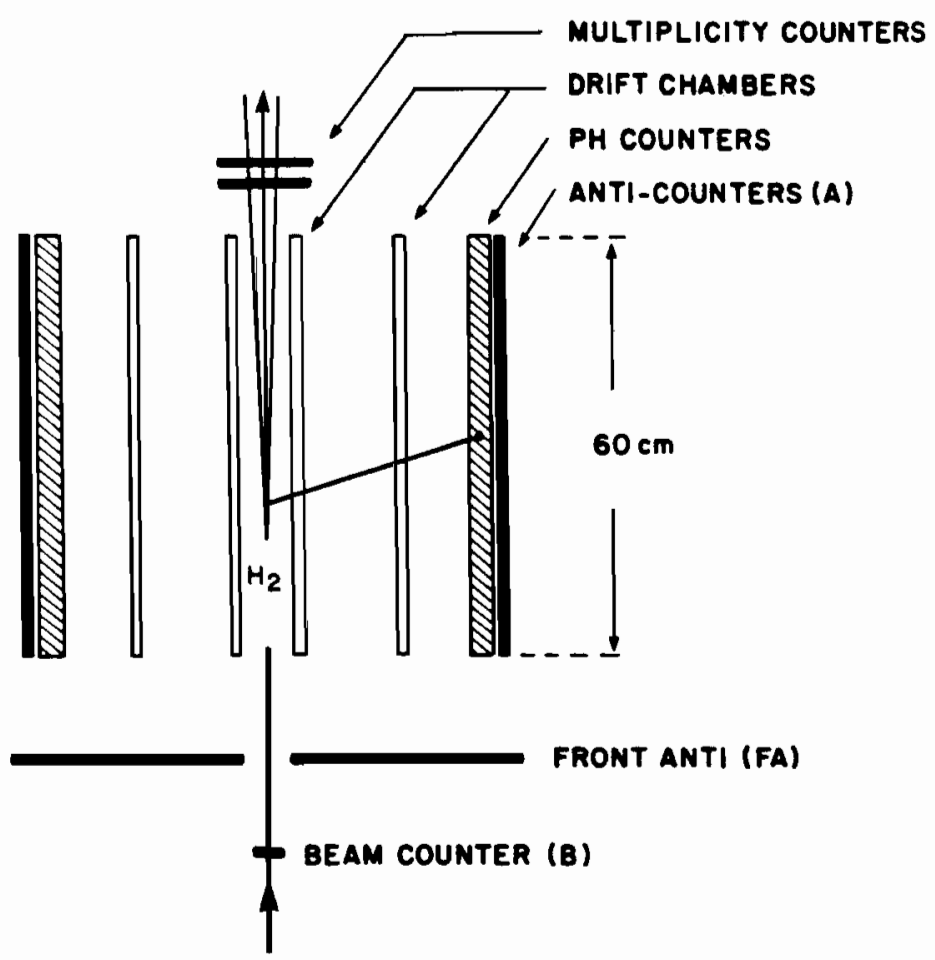

FIG. 1 - Apparatus (plan view, to scale). 
Recoil protons from beam interactions in a hydrogen gas target were detected by drift chambers, which measured the polar angle $\theta$, and were stopped by scintillation counters, which measured the kinetic energy $T$. The missing mass was determined, to an accuracy of $\Delta \mathrm{M}_{x}{ }^{2} / \mathrm{M}_{\mathrm{x}}{ }^{2}= \pm 3 \%$, from $\theta, T$ and the beam momentum, $\mathrm{p}_{\mathrm{o}}$ :

$$
M_{x}^{2}=M_{h}^{2}+2 p_{o} \sqrt{2 m_{p}^{T}}\left(\cos \theta-\sqrt{T / 2 m_{p}}\right)
$$

The charged multiplicities were obtained from the pulse height recorded by two scintillation counters located downstream of the recoil detector. Landau fluctuations were reduced by accepting the smaller of the two normalized pulse heights. Figure 2 shows an example of a pulse height distribution. The curve is a maximum likelihood fit to Landau distributions calculated for each multiplicity.

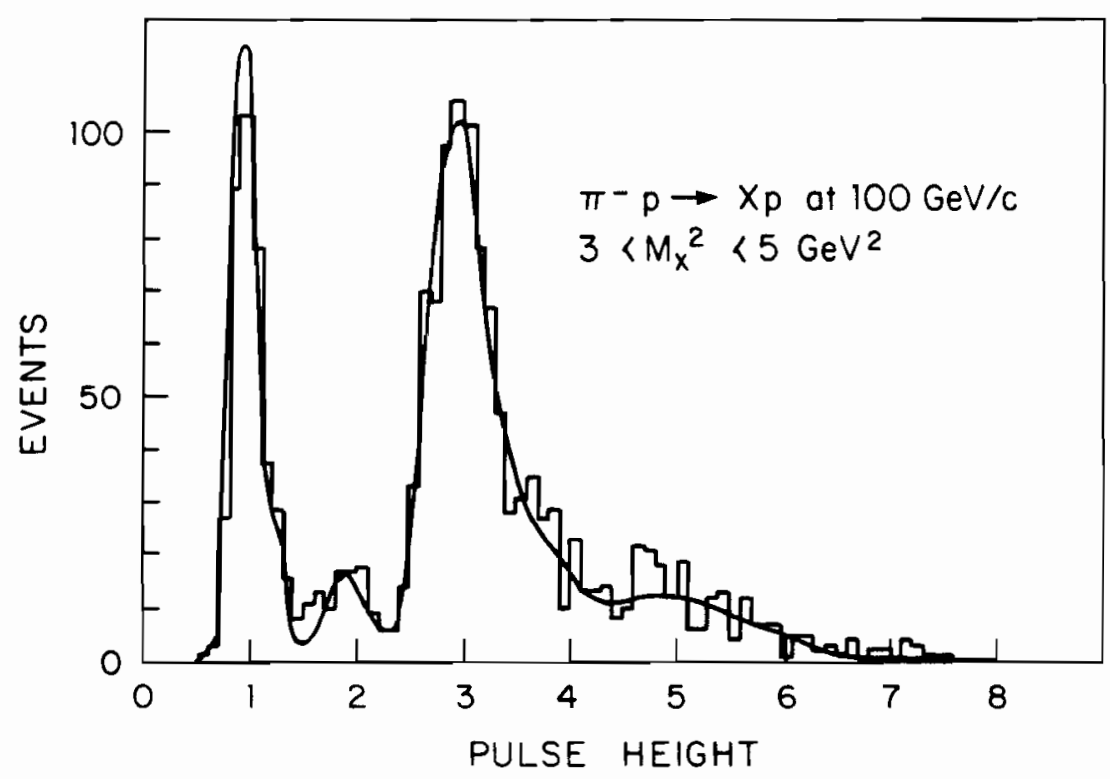

FIG. 2 - Typical multiplicity counter pulse height distribution. For each event, the smaller of the two pulse heights was used.

The raw multiplicities obtained in this manner were corrected for background tracks, for extra (accidental) beam particles and for the acceptance of the multiplicity counters. The background was estimated by using events with negative (unphysical) $M^{2}$ and was subtracted from the topological cross sections at the corresponding positive $M_{x}{ }^{2}$. The subtractions were generally less than $10 \%$. The fraction of events with an extra beam track was measured to be $\$ 10 \%$ by comparing elastic events with charge 2 to those of charge 1. Assuming an isotropic decay distribution in the center of mass of the diffractive state, the calculated average acceptance of the counters is $95 \%$, which agrees well with that estimated from the number of unphysical even charged multiplicity events caused either by an extra beam track or by a track missing the counters. 


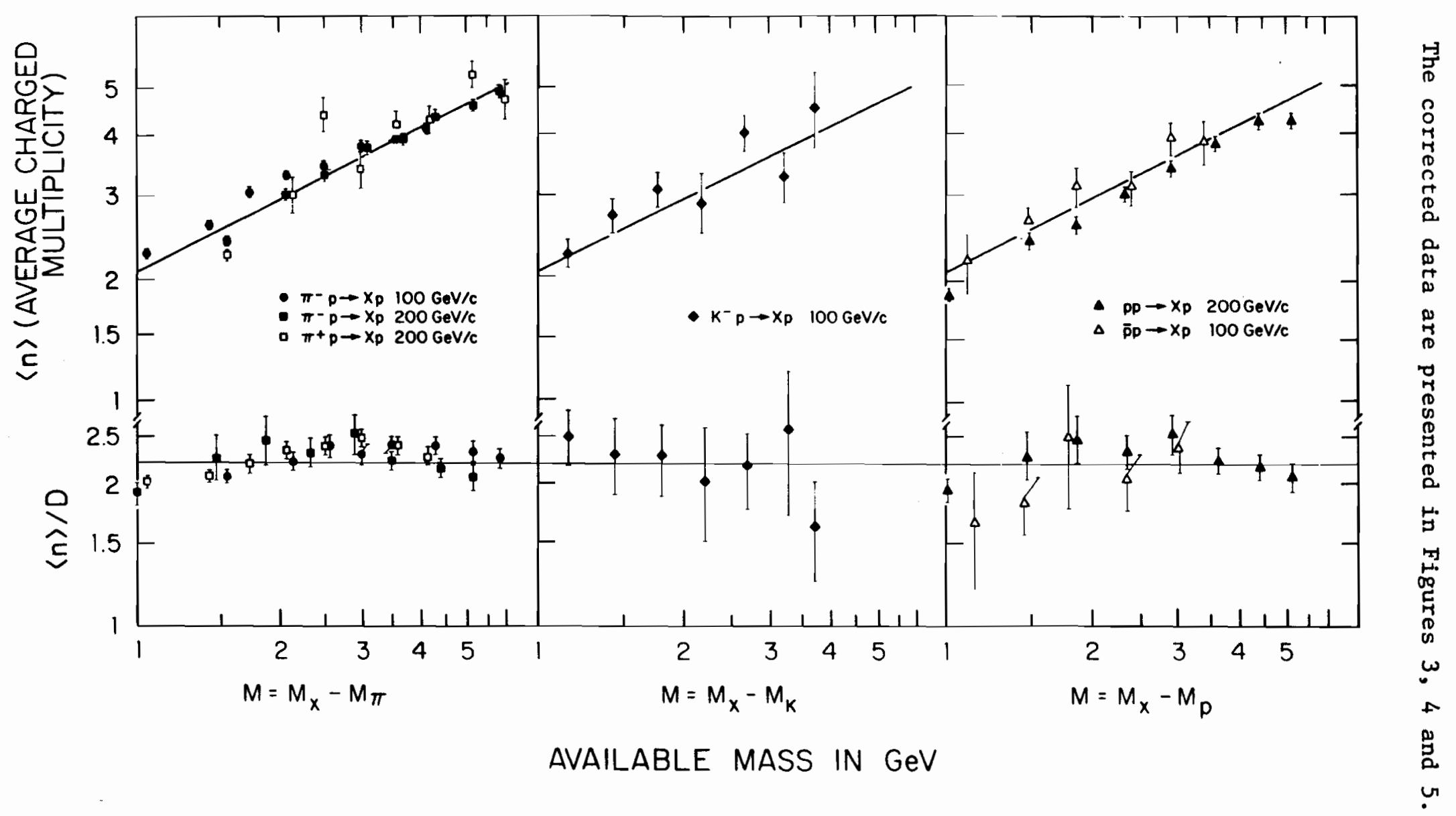

FIG. 3 - Average charged multiplicity and ratio of average to width as a function of available mass. The curves represent $\langle\mathrm{n}\rangle=2.08 \mathrm{M}^{\frac{1}{2}}$ and $\langle\mathrm{n}\rangle / \mathrm{D}=2.2$ as discussed in the text. 


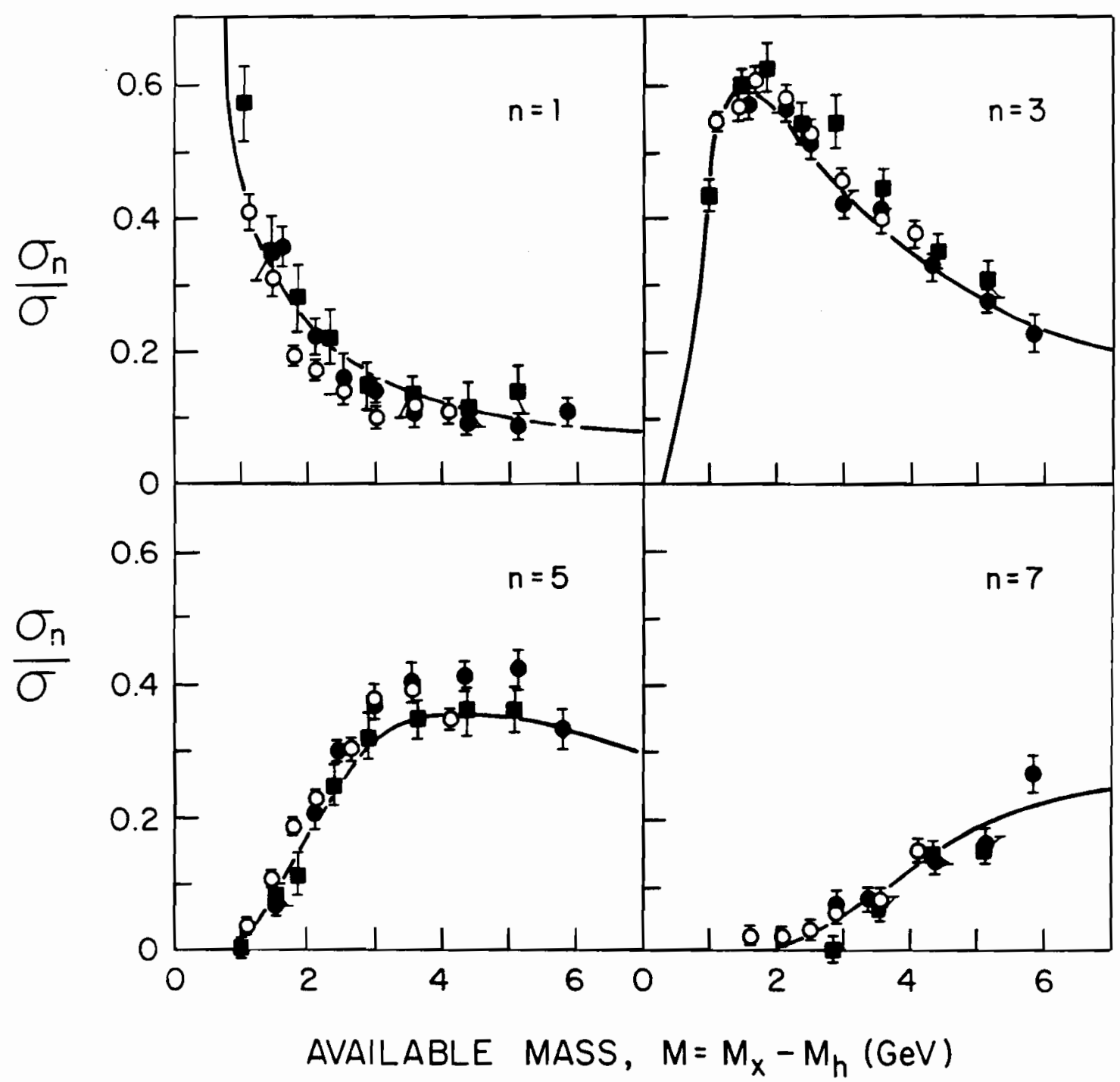

$0 \pi^{-p} 100 \mathrm{GeV} / \mathrm{c} \quad \pi^{-p} 200 \mathrm{GeV} / \mathrm{c} \quad \mathrm{pp} 200 \mathrm{GeV} / \mathrm{c}$

FIG. 4 - Charged multiplicity fractions versus available mass for $h p+X p\left(h=\pi^{-}, p\right)$. 


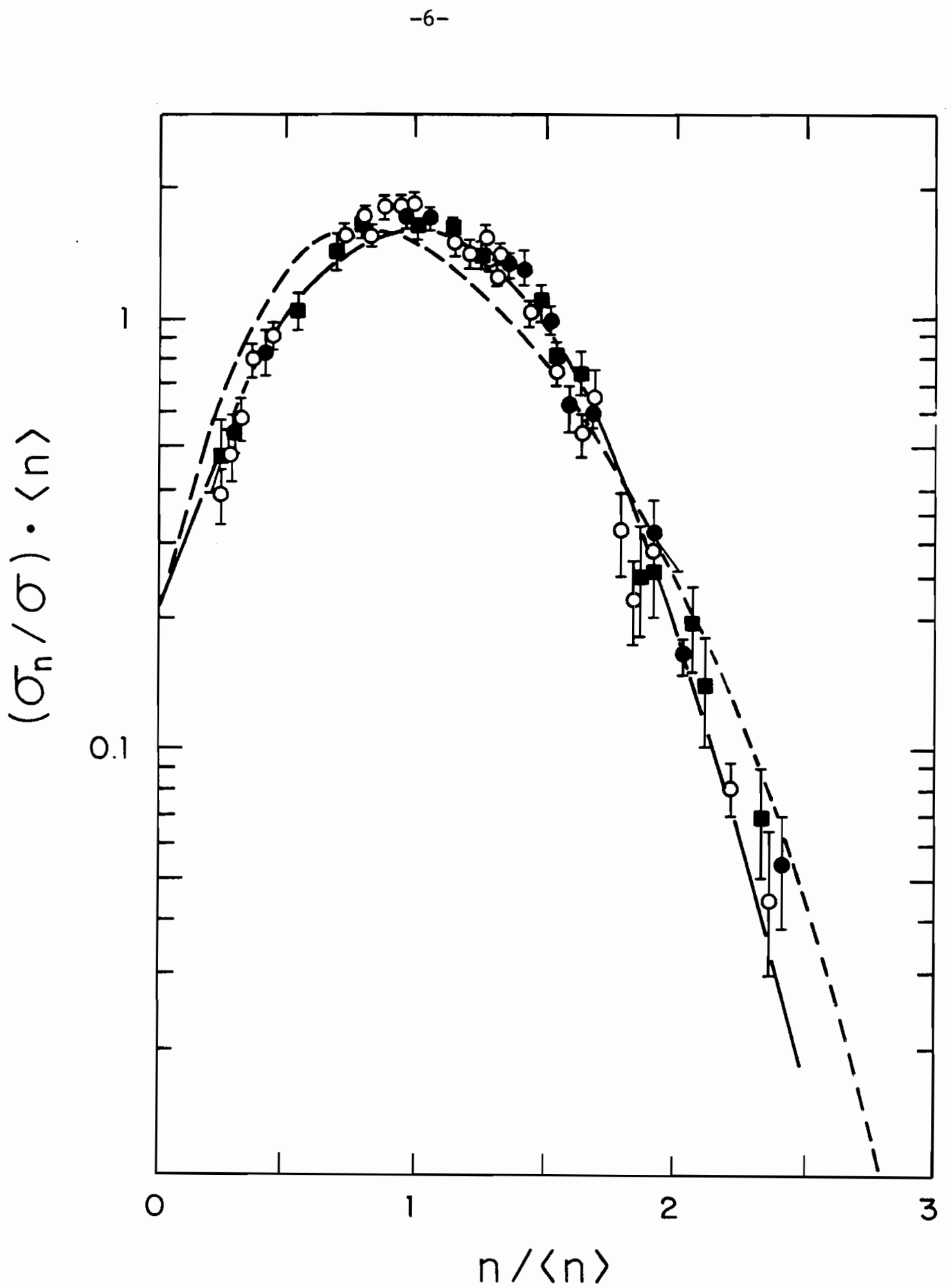

FIG. 5 - The product $\left(\sigma_{n} / \sigma\right) \cdot\langle n\rangle$ versus $n /\langle n\rangle$ for the data presented in Fig. 4. The solid line represents the Gaussian function discussed in the text (Eq. 4). The broken line is from a fit to the inclusive data hp $\rightarrow$ anything (Ref. 4). 
The following features are noted:

(i) Within the statistical accuracy, the multiplicities of all three hadrons are the same when compared at the same mass available for the production of pions, $M=M_{x}-M_{h}$. This would not be true if the comparison were made at the same $M_{x}$.

(ii) The average multiplicity increases with $M$, approximately as $\langle\mathbf{n}\rangle \cong 2 \mathrm{M}^{\frac{1}{2}}$, where $M$ is in $\mathrm{GeV}$, while the ratio $\langle\mathrm{n}\rangle / \mathrm{D}$, where $D=\left(\left\langle n^{2}\right\rangle-\langle n\rangle^{2}\right)^{\frac{1}{2}}$, remains constant at the value of $n 2.2$ (see Fig.3). The function $\langle n\rangle=a+b \ln (M)$ would also provide a good fit to our data but we prefer the power dependence because, as will be shown in Part II, it characterizes the average multiplicity of many processes at higher available energy whereas a simple logarithmic dependence falls too low.

(iii) The ratio of the topological cross section $\sigma_{n}$ to the total diffractive cross section $\sigma$ (see Fig. 4) is described well by the Gaussian function

$$
\begin{aligned}
& \frac{\sigma_{n}}{\sigma}=P_{n}=\frac{2}{\sqrt{2 \pi} D} e^{-\frac{\left(n-n_{0}\right)^{2}}{2 D^{2}}} \\
& n_{0}=2 M^{\frac{1}{2}}(M \text { in } G e V) \\
& n_{0} / D=2
\end{aligned}
$$

The normalization of $P_{n}$ is such that at any value of $M$ the sum of $P_{n}$ over odd values of $n$ is unity to within $\sim 1 \%$. In calculating values of $\langle\mathrm{n}\rangle$ and $\mathrm{D}$ to compare with experimental data, one must sum over positive values of $n$ only. This shifts the average by $\sim 4 \%$ above the value of $n_{0}$ and the width down by $\sim 6 \%$ so that the average multiplicity becomes $\langle\mathrm{n}\rangle=2.08 \mathrm{M}^{\frac{1}{2}}$ and the $\langle\mathrm{n}\rangle / \mathrm{D}$ ratio is increased by $10 \%$ above the value of $n_{0} / D$, to the measured values of 2.2

The form (3) of the probability function suggests that the multiplicity distributions satisfy KNO scaling[3]; i.e., that the product $\mathrm{P}_{\mathrm{n}} \cdot\langle\mathrm{n}\rangle$ is a function of $\mathrm{n} /\langle\mathrm{n}\rangle$ only and not a function of $\mathrm{M}$. Indeed, from $(3 a, b, c)$ it follows that

$$
P_{n} \cdot n_{0}=\sqrt{\frac{8}{\pi}} e^{-2\left(1-n / n_{0}\right)^{2}}
$$

Figure 5 shows $P_{n} \cdot\left\langle n>\right.$ versus $n /<n>$ for the $\pi^{-} p$ and the $p p$ data. Ignoring the small difference between $\langle\mathrm{n}\rangle$ and $\mathrm{n}_{\mathrm{o}}$, one sees that function (4) represents the data well. In contrast to the fully inclusive 
reactions $\mathrm{pp} \rightarrow$ anything and $\pi p \rightarrow$ anything whose $\mathrm{KNO}$ scaling curve ${ }^{[4]}$, also shown in Fig. 5 , is asymmetric and peaks at $n /<n>\cong 0.8$, the distribution for the diffractive data is symmetric about the peak at $\mathrm{n} /\langle\mathrm{n}\rangle=1$.

Our results for $\langle\mathrm{n}\rangle$ and $\langle\mathrm{n}\rangle / \mathrm{D}$ agree well with previous measurements[5]. However, the multiplicity distributions obtained in these measurements $[5,6]$ are inconsistent with ours in that they follow an asyminetric scaling curve similar to that of the inclusive case. We attribute the inconsistency to their use of large $\mathrm{M}_{\mathrm{x}}{ }^{2}$ bins needed for adequate statistics in presenting the distributions, for example $0<\mathrm{M}^{2}<32(\mathrm{GeV} / \mathrm{c})^{2}$, which integrates the data over large variations in average and width and distorts the distributions.

In summary, we find that the charged multiplicity distributions of high mass diffractive pion, kaon and nucleon states follow a universal Gaussian function that depends only on the available mass $M(G e V)$, has a peak that varies with $M$ as $n_{0} \cong 2 M^{\frac{1}{2}}$, and a peak to width ratio $\mathrm{n}_{\mathrm{o}} / \mathrm{D} \cong 2$.

\title{
PART II
}

\author{
INCLUSIVE HADRONIC CHARGED MULTIPLICITIES \\ and \\ UNIVERSALITY OF CHARGED MULTIPLICITY DISTRIBUTIONS
}

It is well known $[4,7,8]$ that charged multiplicity data of inclusive hadronic reactions do not agree with Eq. 3. For example, for pp $\rightarrow$ anything $[4,7,8,9]$, the increase of the average multiplicity with energy is slower than $2 s^{\frac{1}{4}}$ (see Fig. $6 a$ ), the $\langle n\rangle / D$ ratio decreases as the energy increases (see Fig. 6b), and the KNO distribution of the data is asymmetric about $n \mid\langle n\rangle=1$ (see Fig. 7) in disagreement with Eq. 4. On the other hand, data on $\mathrm{e}^{+} \mathrm{e}^{-} \rightarrow$ anything are consistent $[10]$ with $\langle\mathrm{n}\rangle=2 \mathrm{~s}^{\frac{1}{4}}$ (see Fig. 8) and their KNO scaling curve is symmetric [11].

In this part, we analyze the inclusive $\mathrm{pp} \rightarrow$ anything charged multiplicities, recognizing that they derive from two distinct sectors of the inelastic cross section: the diffractive component, for which the available mass is $M=M_{x}-M_{p}$, and the non-diffractive "hard core" for which $M=\sqrt{s}-2 M_{p}$. We find that Eq. 3, which describes well the diffractive multiplicities, also provides a good description of the multiplicities of the hard core. After an examination of data from several other reactions, we come to the conclusion that the distribution represented by Eq. 3 is universal, describing to a good approximation all known hadronic charged multiplicities up to and including ISR energies. This suggests that gluons may play an important role in the hadronization process. 


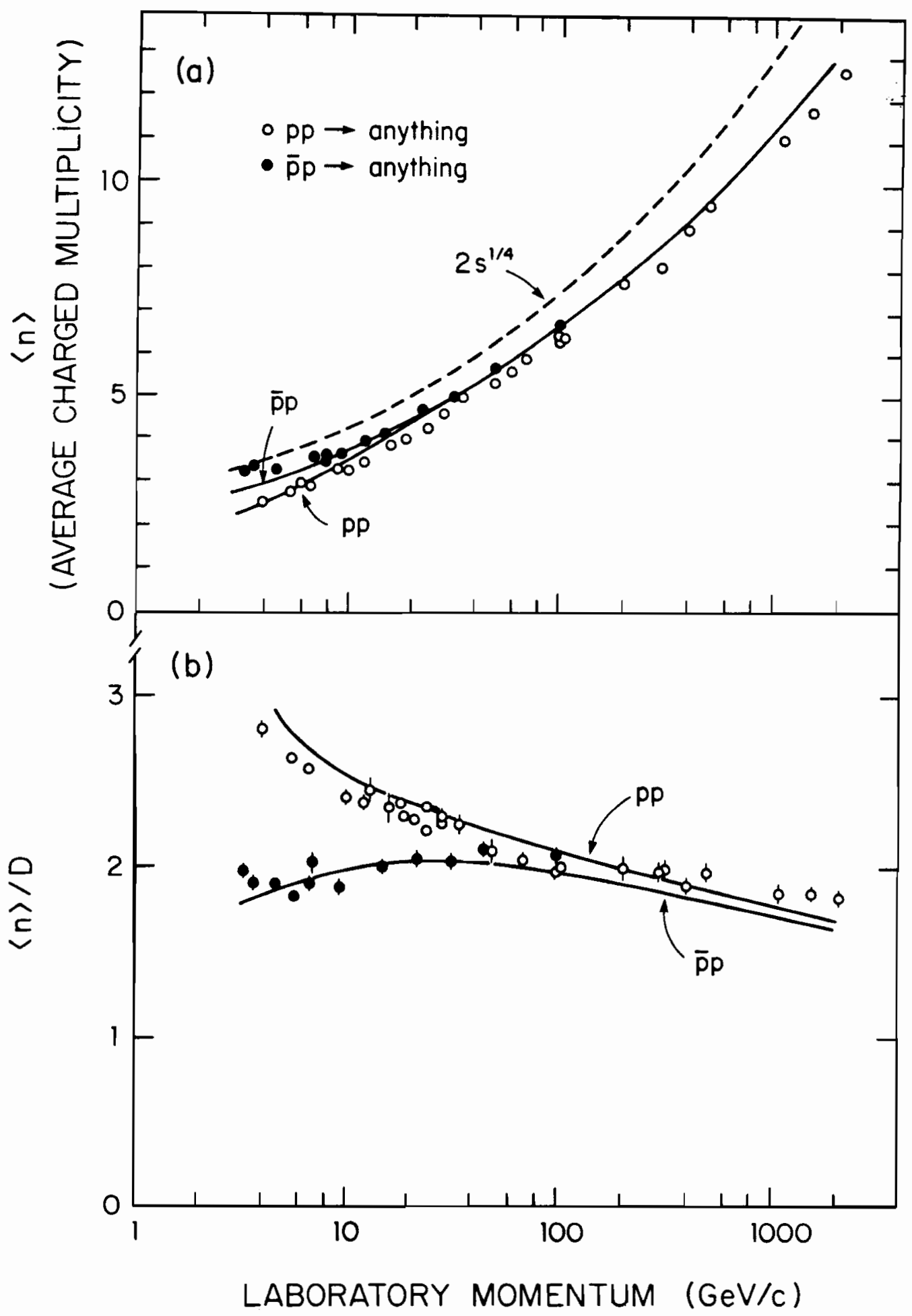

FIG. 6 - The average charged multiplicity and the ratio of the average to the width as a function of $\mathrm{P}_{1 \mathrm{ab}}$ for $\mathrm{pp} \rightarrow$ anything and $\bar{p} p \rightarrow$ anything. The solid curves were calculated using Eqs. 6 and 7 in the text. 


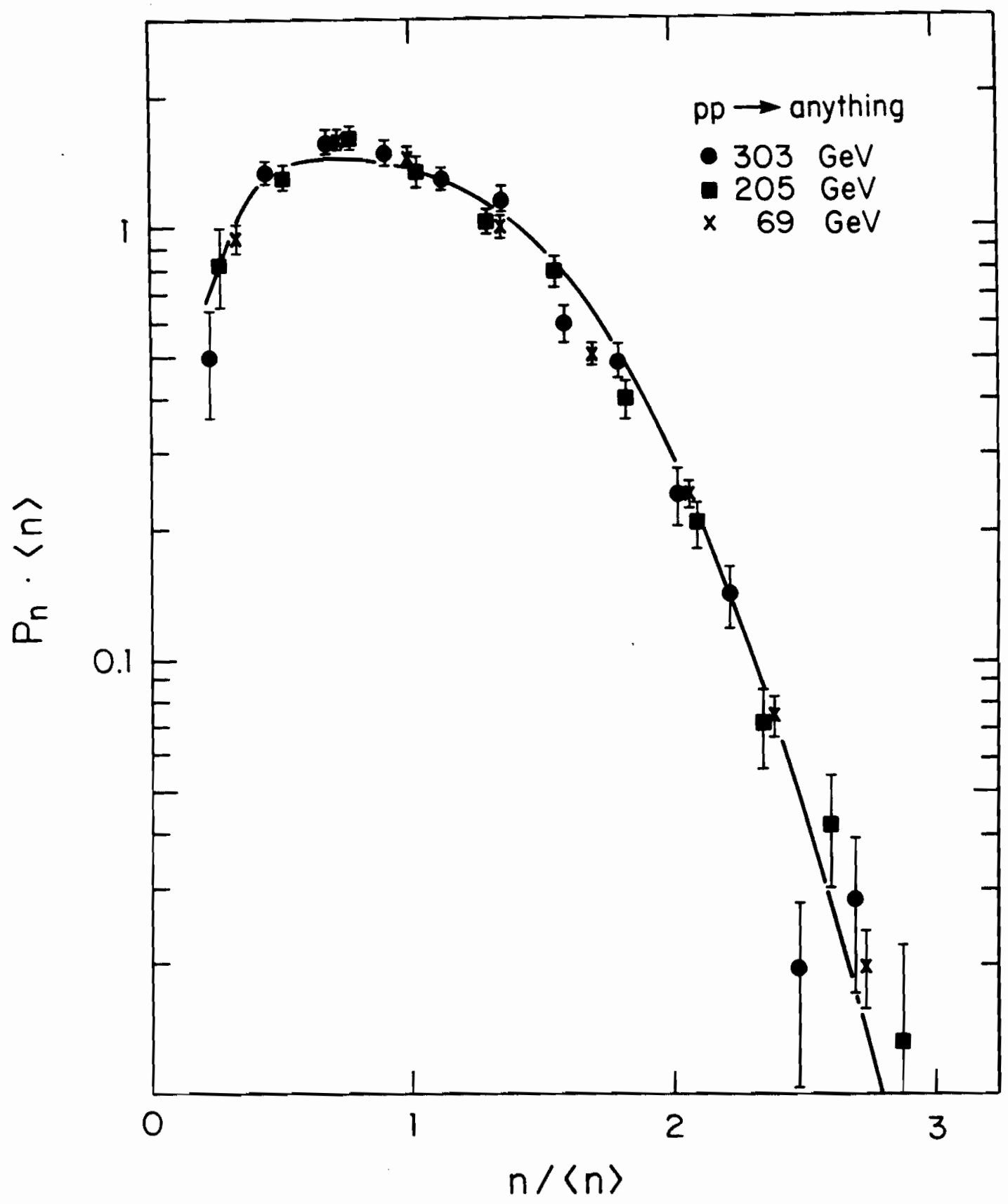

FIG. 7 - The product $P_{n} \cdot\langle n\rangle$ versus $n /\langle n\rangle$ for charged particles in $p p+$ anything. The curve was calculated using Eq. 6 in the text. 


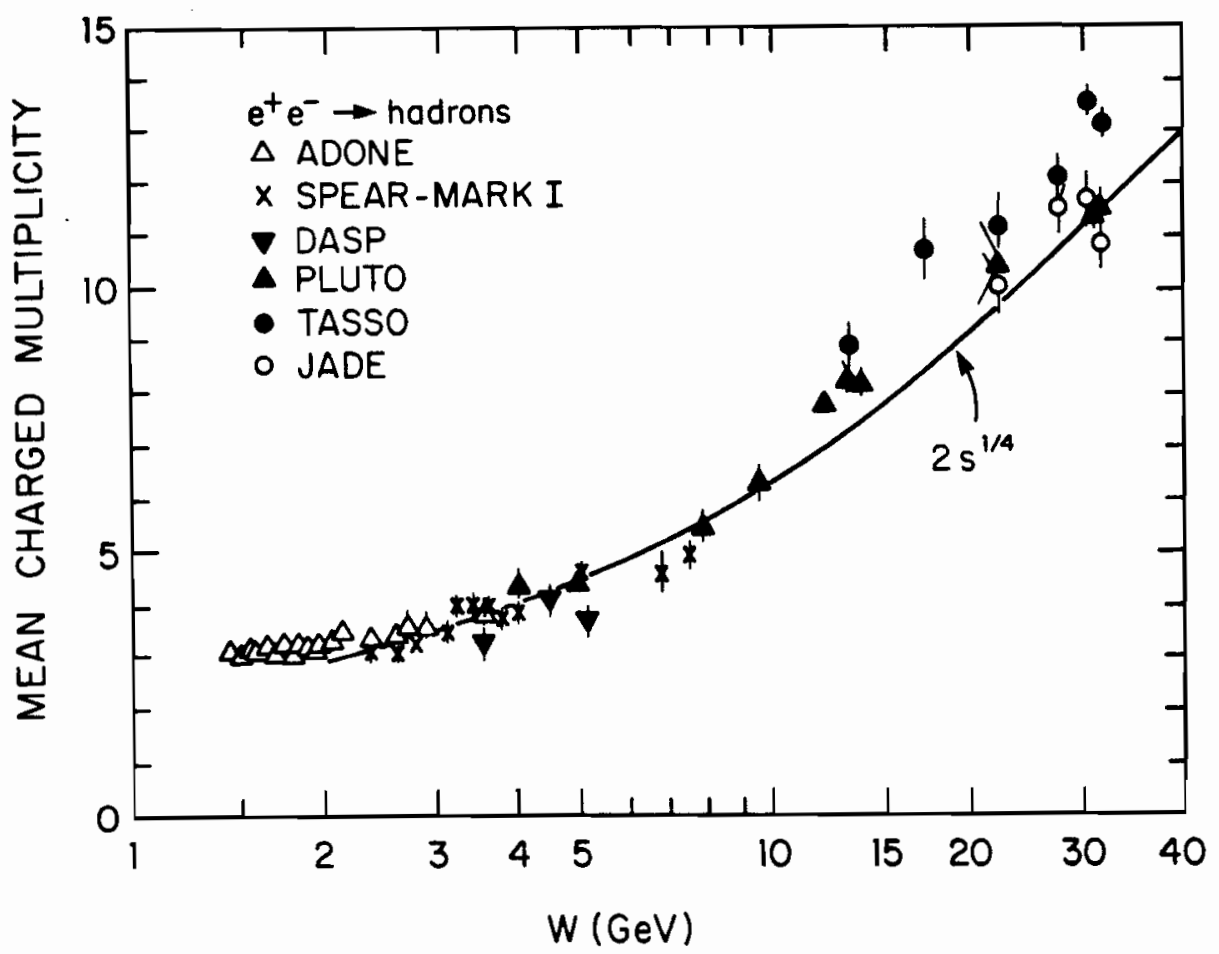

FIG. 8 - Mean charged multiplicity versus available energy for $\mathrm{e}^{+} \mathrm{e}^{-} \rightarrow$ anything.

Small differences in the multiplicities between certain reactions, such as between PP and $\overline{\mathrm{PP}}$, which cannot be explained by our procedure of applying Eq. 3 at the appropriate available mass of each identifiable component of the inelastic cross section, may then be attributed to the difference in the quark content of the initial states.

The total, the elastic, and the inelastic pp cross sections are shown in Figure 9. The inelastic cross section is consistent [12] with being composed of a hard core, $\sigma_{0}=26.3 \mathrm{mb}$, and a diffractive component, $\sigma_{\mathrm{D}}$, which consists of the contribution of single diffraction dissociation, $2 \sigma_{\mathrm{SD}}$, and that of double diffraction dissociation, $\sigma_{\mathrm{DD}}$ :

$$
\sigma_{i}=\sigma_{0}+2 \sigma_{S D}+\sigma_{D D}
$$

In calculating charged multiplicity distributions, we assume that the inelastic cross section in excess of $\sigma_{0}$ is all due to single diffraction dissociation with a $1 / \mathrm{M}^{2}$ mass dependence. The double diffractive process which becomes important only at very high energies, is approximated well by this assumption, since there is only a small probability for both diffractive masses to be large. Multiplicity distributions for pp are then generated using Eq. 3 separately for the diffractive and non-diffractive components at the appropriate available mass: 


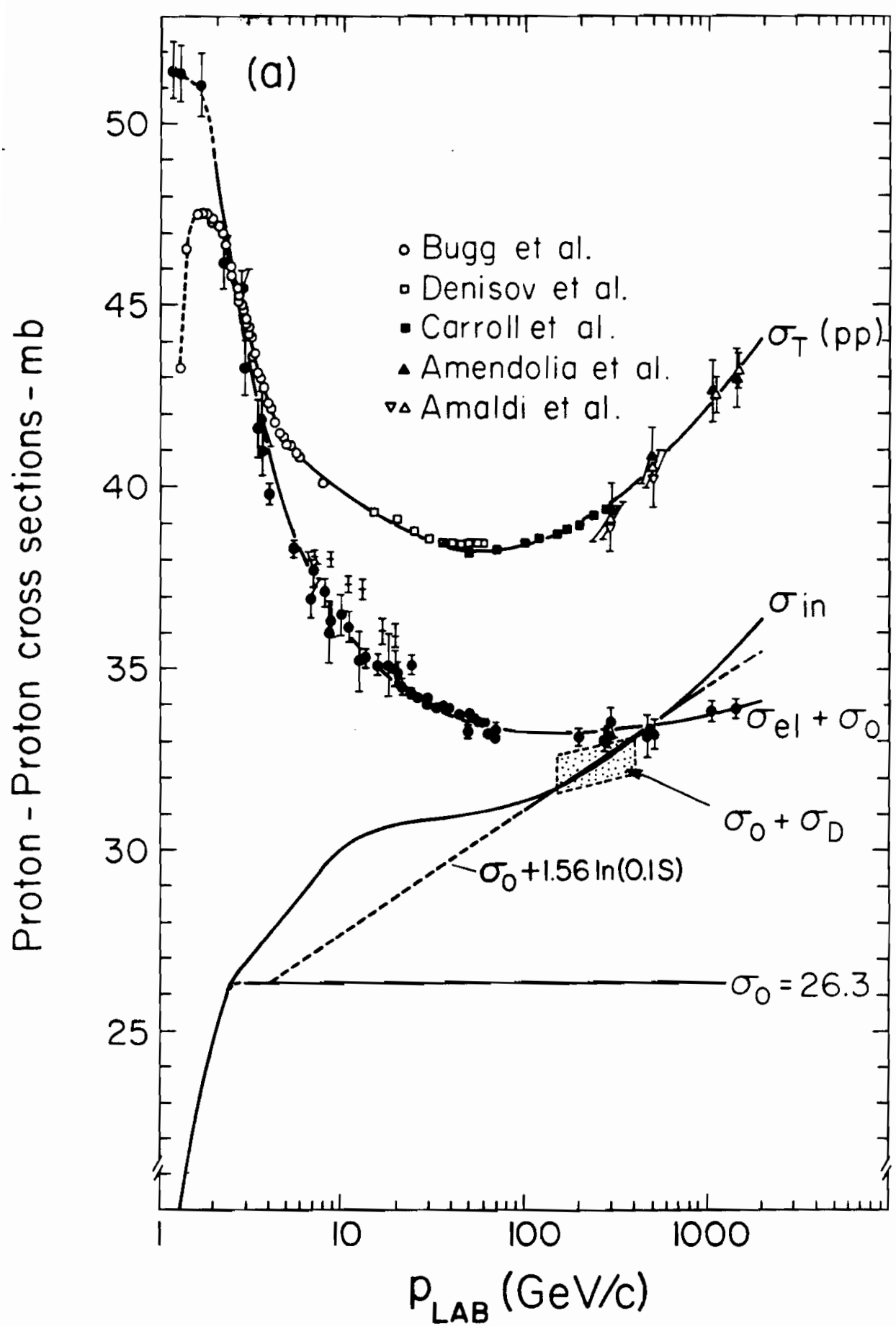

FIG. 9 - The total, the elastic and the inelastic pp cross sections (from Ref. 12). 


$$
\operatorname{P}_{n+2}=\frac{1}{\sigma_{i}}\left[\sigma_{0} P_{n+2}\left(\sqrt{s}-2 M_{p}\right)+\frac{\sigma_{D}}{\ln (0.1 s)} \int_{1}^{0.1 s} \frac{1}{M_{x}{ }^{2}} P_{n+1}\left(M_{x}-M_{p}\right) d M_{x}{ }^{2}\right]
$$

The average multiplicity, the $\langle n\rangle / D$ ratio and the KNO distribution calculated using this equation are in good agreement with the data, as shown in Figures 6 and 7 .

For $\mathrm{pp}$, we add the annihilation cross section, $\bar{\sigma}=\sigma_{\mathrm{T}}(\overline{\mathrm{p}} \mathrm{p})-\sigma_{\mathrm{T}}(\mathrm{pp})$, to the PP inelastic cross section and use the distribution

$$
P_{n}=\frac{1}{\sigma_{i}}\left[\sigma_{0} P_{n}\left(\sqrt{s}-2 M_{p}\right)+\frac{\sigma_{D}}{\ln (0.1 s)} \int_{1}^{0.1 s} \frac{1}{M_{x}{ }^{2}} P_{n-1}\left(M_{x}-M_{p}\right) d M_{x}{ }^{2}+\bar{\sigma}_{n}(\sqrt{s})\right]
$$
where the second term is not included for $n=0$. The $\langle n\rangle$ and $\langle n\rangle / D$
calculated using this distribution function agree well with the data $[7,13]$ (see Fig. 6). However, at high energies, there remains a small but significant difference between the average $\mathrm{pp}$ and $\overline{\mathrm{p}} \mathrm{p}$ multiplicities which is not predicted by the equations given above. If this difference were due to the annihilation cross section, its average multiplicity would have to be larger than $2 \mathrm{~s}^{\frac{1}{4}}$ by more than $50 \%$. We prefer the interpretation that this disparity arises from a small difference in the $\mathrm{pp}$ and $\overline{\mathrm{p}} \mathrm{p}$ hard cores and that the annihilation multiplicities follow the distribution given by Eq. 3 .

A similar analysis of $p p+$ anything in terms of diffractive and nondiffractive components was performed previously[14] and led to the same conclusions about the multiplicities of the hard core. More recently, an ISR experiment [15] which removed leading particle effects obtained similar results. Our contribution in this area is that we have demonstrated that the same function that describes the multiplicity distribution of diffractive high mass states also describes the $\mathrm{pp}$ and $\overline{\mathrm{p}} \mathrm{p}$ hard cores and $\overline{\mathrm{p}} \mathrm{p}$ annihilation.

By applying the same ideas to $\pi^{ \pm} p$ reactions, treating the non-diffractive inelastic cross section as a hard core with available mass $M=\sqrt{s}-M$, we have obtained good agreement of our calculated $\langle\mathrm{n}\rangle$ and $\langle\mathrm{n}\rangle / \mathrm{D}$ with existing data $[7,9,16]$ (see Fig. 10). We have also investigated the reactions $\nu_{\mu}+p \rightarrow \mu^{-}+x^{+}$and $\bar{\nu}_{\mu}+p \rightarrow \mu^{+}+x^{\circ}$. Again, the multiplicity distributions reported for these reactions $[17,18]^{\circ}$ are in good agreement with our predictions (see Figs. 11 and 12). In particular, the higher average multiplicity and $\left\langle n>/ D\right.$ ratio[17] of $x^{++}$relative to that of $x^{0}$ arise naturally as a consequence of summing over $n=2,4,6 \ldots$ for $x^{++}$ and $n=0,2,4 \ldots$ for $x^{\circ}$. A determination[19] of the average multiplicity of the state $X$ in $P P \rightarrow \mu^{+} \mu^{-} X$ at ISR is also consistent with $2 s^{\frac{1}{4}}$ behavior (see Fig. 13). 


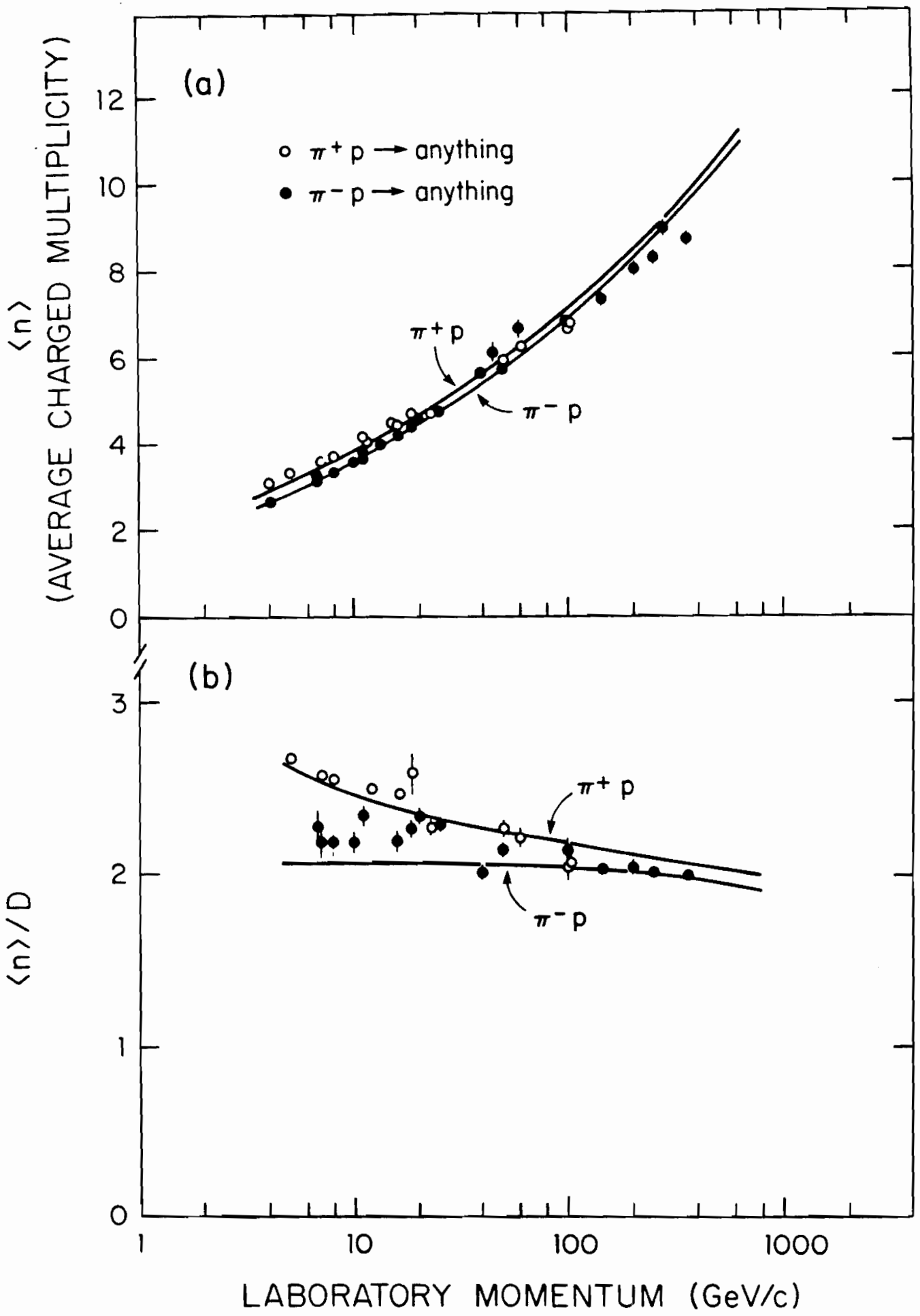

FIG. 10 - Charged $\langle n\rangle$ and $\langle n\rangle / D$ for $\pi^{ \pm} p+$ anything. The curves are predictions based on Eq. 3 as discussed in the text. 

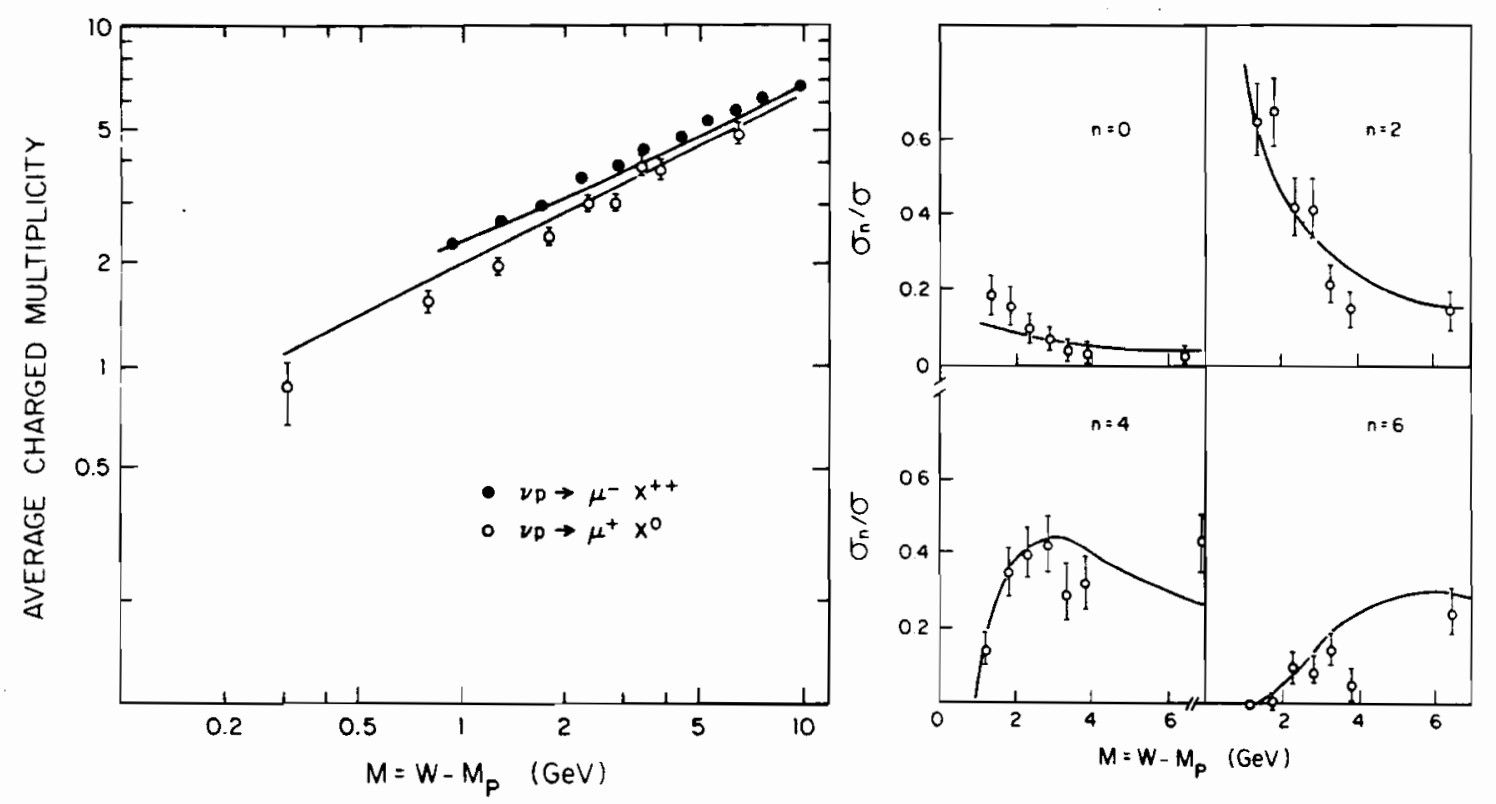

FIG. 11 - Charged $\langle\mathrm{n}\rangle$ versus available energy for $\nu_{p} \rightarrow \mu^{-} x^{++}$and $\bar{\nu} p \rightarrow \mu^{+} x^{\circ}$. The curves are predictions based on Eq. 3 as discussed in the text.

FIG. 12 - Charged multiplicity fractions for $\bar{v}_{p} \rightarrow \mu^{+} x^{\circ}$. The curves are from Eq. 3 in the text.

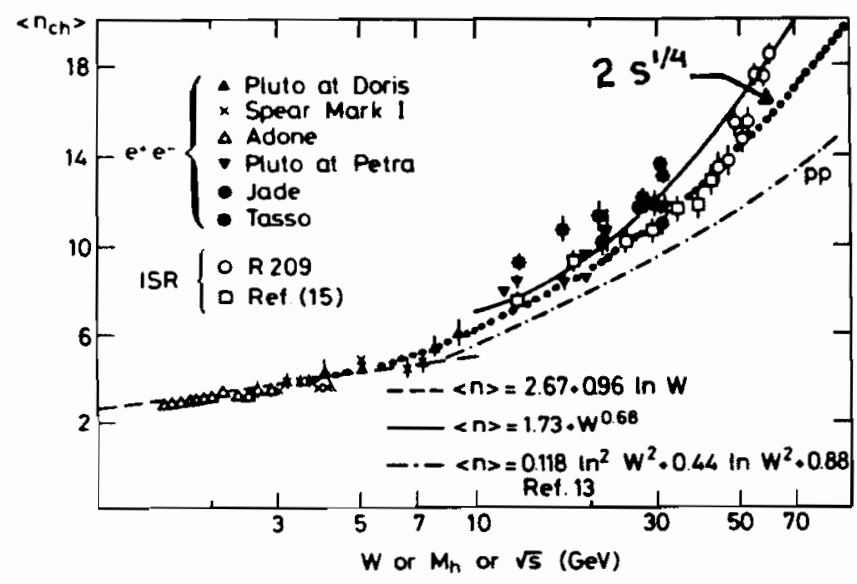

FIG. 13 - Average multiplicity versus available energy (from Ref. 19). 
Recently, two $\bar{p}$ experiments being performed at the CERN SPS Collider at $\sqrt{\mathrm{s}}=540 \mathrm{GeV}$ reported the values $3.9 \pm 0.3^{[20]}$ and $3.0 \pm 0.1^{[21]}$ for $\mathrm{d}<\mathrm{n}>/\left.\mathrm{dn}\right|_{n=0}$, the average charged multiplicity per unit rapidity in the central region. At ISR energies, the values of the rapidity plateau are lower. Typically, at $\sqrt{\mathrm{s}}=30.8$ and $62.8 \mathrm{GeV}, \mathrm{d}<\mathrm{n}>/\left.\mathrm{d} n\right|_{n=0}=1.67$ and 2.03 , respectively $[8]$. Since the rapidity interval increases with energy as $\ell$ ns and the charged multiplicity as $s^{\frac{1}{4}}$, we expect $\mathrm{d}<\mathrm{n}>\mathrm{d} \eta$ to vary as $\mathrm{Cs}^{\frac{1}{4}} /$ lns. Setting $\mathrm{C}=2.1$ gives $1.71,2.00$ and 3.91 at $\sqrt{\mathrm{s}}=30.862 .8$ and $540 \mathrm{GeV}$, in excellent agreement with the ISR measurements and the SPS Collider result of Ref. 20 (see Fig. 14).

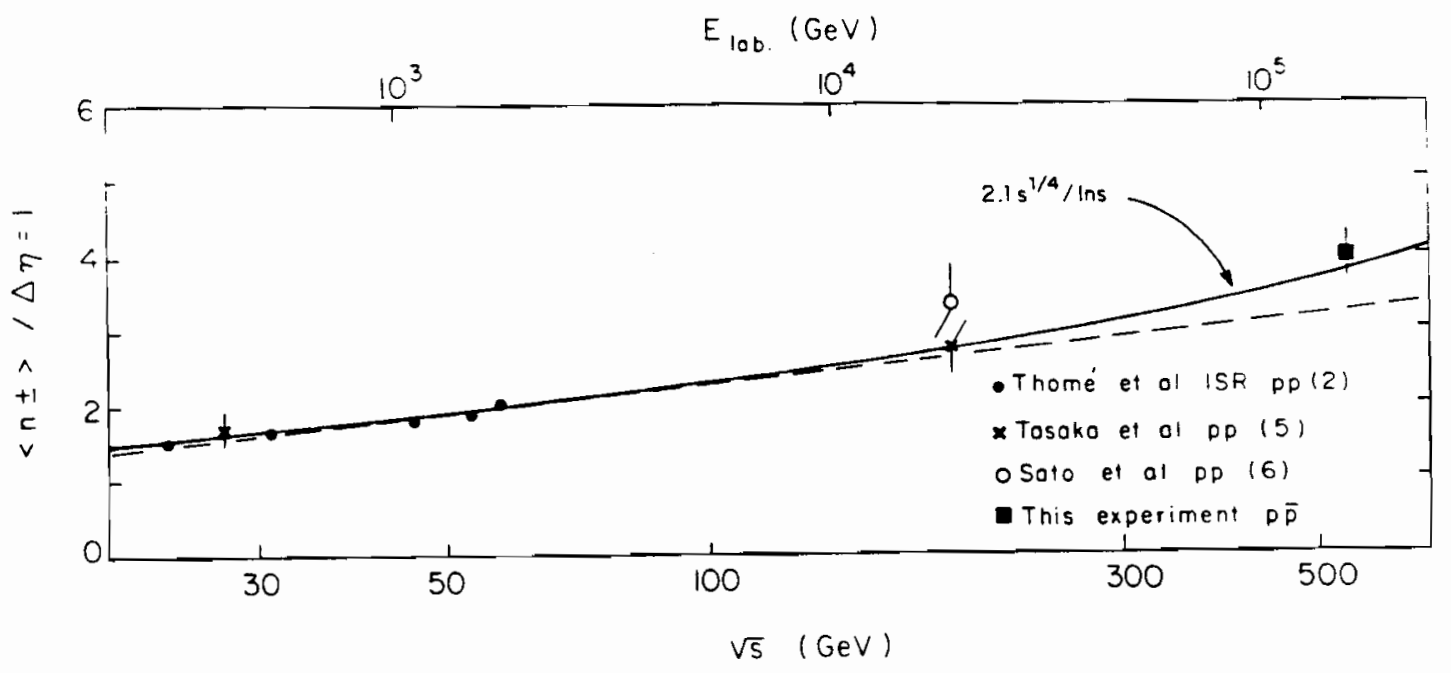

FIG. 14 - Average charged multiplicities in the central region (from Ref. 20). The solid curve is our prediction as discussed in the text.

\section{CONCLUSION}

In conclusion, we find that the distribution represented by Eq. 3 provides a good description of the charged multiplicites not only of high mass diffractive states but also of a wide variety of other hadronic states where the available mass for pionization can be identified. This universality of the multiplicity distribution implies that the quark content of the dissociating state does not play an important role in determining the multiplicity and therefore it must be that the gluons dominate the process of hadronization. 


\section{REFERENCES}

1. R.L. Cool et al., Phys. Rev. D24, 2821 (1981).

2. R.L. Cool et al., Phys. Rev. Lett. 47, 701 (1981).

3. 2. Koba, H.B. Nielson and P. Olesen, Nuc1. Phys. B40, 317 (1972).

4. P. Slattery, Phys. Rev. D7, 2073 (1973).

5. $\pi \mathrm{p}$ : F.C. Winkelmann et a1., Phys. Rev. Lett. 32, 121 (1974).

Pp : F.T. Dao et al., Phys. Lett. 45B, 399 (1973); S.J. Barish, et al., Phys. Rev. Lett. 31, 1080 (1973); J.W. Chapman et al., Phys. Rev. Lett. 32, 257 (1974); and M.G. Albrow et a1., Nucl. Phys. B102, 275 (1976).

$\overline{p p}$ : F. Grard et a1., Phys. Lett. 59B, 409 (1975); C.P. Ward et al., Nucl. Phys. B153, 299 (1979).

6. S. Barshay et al., Phys. Rev. Lett. 32, 1390 (1974).

7. E. DeWolf et al., Nuc1. Phys. B87, 325 (1975); E. Albini et al., Nuovo Cimento 32, 101 (1976).

8. W. Thomé et al., Nuc1. Phys. B129, 365 (1977).

9. C. Bromberg et al., Phys. Rev. D15, 64 (1977); W.M. Morse et al., Phys. Rev. D15, 66 (1977); A. Firestone et al., Phys. Rev. D10, 2080 (1974); S. Barish et al., Phys. Rev. D9, 2689 (1974); V.V. Ammosov et al., Nuc1. Phys. B58, 77 (1973).

10. ADONE : C. Bacci et al., Phys. Lett. 86B, 234 (1979). SPEAR-MARK I : J.L. Siegrist, Ph.D. Thesis SLAC-225 UC-34d (1980). DASP : R. Brandelik et al., Nucl. Phys. B148, 189 (1979).

PLUTO : Ch. Berger et al., Phys. Lett. $78 \overline{\mathrm{B}, 176}$ (1978).

TASSO : R. Brandelik et al., Phys. Lett. $89 \mathrm{~B}, 418$ (1980); ibid, 94B, 444 (1980).

JADE : W. Bartel et al., Phys. Lett. 88B, 171 (1979).

11. Ch. Berger et al., Phys. Lett. 95B, 313 (1980).

12. K. Goulianos, Phys. Rev. D14, $1 \overline{445}$ (1976).

13. D.E. Zissa et al., Phys. Rev. D21, 3059 (1979); R.E. Ansorge et al., Phys. Lett. 59B, 299 (1975).

14. K. Fialkowski and H.I. Miettinen, Phys. Lett. 43B, 61 (1973).

15. M. Basile et a1., Phys. Lett. 95B, 311 (1980).

16. P.J. Hays et a1., Phys. Rev. $\overline{D 23}, 20$ (1980); A. Firestone et al., Phys. Rev. D14, 2902 (1976); D. Fung et a1., Nucl. Phys. B102, 386 (1976).

17. P. Allen et al., Nucl. Phys. B181, 385 (1981).

18. M. Derrick et al., Phys. Rev. D17, 17 (1978).

19. D. Antreasyan et al., CERN-EP/81-116.

20. UAI Collaboration, CERN-EP/81-155.

21. UA5 Collaboration, CERN-EP/81-152. 